\title{
Comment on: "A Multinational European Study of Patient Preferences for Novel Diagnostics to Manage Antimicrobial Resistance"
}

\author{
J. P. Hays ${ }^{1}$ \\ (c) Springer Nature Switzerland AG 2020
}

\section{Dear Editor,}

I read with interest the work of Mott et al., published in Applied Health Economics and Health Policy in 2020 [1]. In this publication, the authors conclude that "Patients in different European countries do not have the same preferences for the attributes of diagnostic tests to manage antimicrobial resistance (AMR) in primary care."

In this respect, and in respect to the comment "Considering patient preferences when designing diagnostic tests is important because individuals' preferences could directly influence their uptake of such tests, yet to date no studies have explored this issue.", I would like to draw the authors' attention to an article published on a related theme from 2013, namely "Perceptions of point-of-care infectious disease testing among European medical personnel, pointof-care test kit manufacturers, and the general public" [2], which was part of a European Union (EU)-funded FP7 project (TEMPOtest-QC_-Grant agreement ID 241742) [3]. I would also like to draw on my own (albeit non-economicsbased) experience in this area [4-7].

Point-of-Care Although not mentioned, I think that the article by Mott et al. should perhaps have specifically used the term 'Point-of-Care' or 'Point-of-Need' (POC) diagnostics, as the research performed is focussed on individuals that had been patients prescribed antibiotics within the last 2 years ("Medical professionals were excluded from

This comment refers to the article available at https://doi. org/10.1007/s40258-019-00516-0.

An author's reply to this comment is available at https://doi. org/10.1007/s40258-020-00573-w

J. P. Hays

j.hays@erasmusmc.nl

1 Department of Medical Microbiology and Infectious Diseases, Erasmus University Medical Centre (Erasmus MC), Rotterdam, The Netherlands participating."), and this focal group may seldom have a say in the choice of diagnostic that is to be used to treat them. An exception to this assertion is POC testing, where the patient privately purchases their own diagnostic test and therefore actively contributes to the diagnostic purchasing process. Therefore, I would suggest that the statement "Patient preferences are important in determining whether diagnostic tests are successful in practice" may have little relevance outside of POC testing (see also 'Expectations').

Expectations The authors write "Patient perceptions are important when it comes to the use and misuse of antibiotics as the overuse of antibiotics is shaped significantly by physicians' desire to satisfy patient demand. It has been illustrated that parental expectations around antimicrobial prescribing drives clinicians' prescription behaviour, and it has been suggested that joint physician-patient (or physiciancarer) level decision making needs to be addressed in order to tackle the misuse of antibiotics." Basically, the first statement relates to certain patients demanding antibiotics from physicians without sufficient evidence to support their actual prescription. I am not convinced that talking with patients, or offering them a diagnostic test, is a feasible option here. This opinion is based on the time constraints involved in the doctor-patient consult and the possible extra costs incurred in performing a diagnostic test (which in any case may not actually change the patient's mind). A better option perhaps would be to offer the patients a tangible alternative 'therapy', but not antibiotics. In the article "'Out-of-the-box' thinking to help stop the spread of antibiotic resistance" [8], I suggested the use of multivitamins as a first-line response to patients that insist on receiving a course of antibiotics. This would be less time consuming, probably more cost effective than using a diagnostic test and, importantly, perhaps better appreciated by the patient.

Costs The authors state that "Whilst many studies examine willingness to pay, cost was not included as an attribute in the discrete choice experiment (DCE) as it was not deemed appropriate in this context (most of the countries 
studied have largely publicly funded health care systems).", which appears to contradict the focal group of the research; that is, individuals that had been patients prescribed antibiotics within the last 2 years. In a world ruled by economics, it would be difficult to justify the use of a diagnostic to manage infection if the financial costs of prevention, treatment and disease are not known (this calculation includes the healthcare costs associated with primary care physician budgets). By omitting medical professionals, it appears that the authors necessarily focus on potential end users, that is, the group that would be buying and using these kind of tests-the patients themselves. Further, private healthcare within publicly funded healthcare systems is not forbidden and many end users may consider buying 'off-the-shelf' or 'off-the-internet' diagnostic tests if they feel that they are not receiving the most appropriate care within publicly funded healthcare systems. This means that costs are likely to be a major factor in the choice of diagnostic by end users, and as such most certainly should be considered by manufacturers of such diagnostics. From our own research, it appears that the price of a (POC) diagnostic is indeed a consideration in the potential use of such diagnostics by the general public. Importantly, not all members of the general public expect to receive free diagnostic testing and would be prepared to pay between $€ 5$ and $€ 25$ (Table 3-2011/2012 prices [2]) for a single diagnostic test. The authors state "Additionally, it has been shown that inclusion of a cost attribute can affect choice behaviour in DCEs, resulting in different relative preferences and an increase in error variance." However, I am not sure that this is a valid criterion not to include costs in the analysis. Perhaps the limitation lies with the DCE analysis as a technique, rather than the choice of 'cost' as a preference variable?

Confidence One of the key points mentioned by the authors is that "confidence in the test result was the most important attribute for patients in some countries". Most diagnostics are performed by trained clinicians or nurses, so I would be surprised if a patient asks their healthcare worker about the healthcare workers' confidence in the result. However, I can imagine that if the result goes against patient expectations-leading to, for example, a positive HIV result, or a decision involving non-prescription of antibiotics - then the patient may question the result. Also, whether the healthcare worker has information available regarding a diagnostics (relative) performance (e.g., specificity, sensitivity, etc.) is another issue. Finally, even if an answer is provided such as "The error rate is 1 in 1000 ", patients may still believe that they are the 1 out of 1000 patients that has received an incorrect result.
In conclusion, Mott et al., have produced a valuable internationally relevant publication with some important recommendations for AMR diagnostics. However, there appear to be some potential caveats in the methodology of the research that may influence the general applicability of the findings. In any case, we agree that a 'one size fits all' approach is likely not feasible for (POC) diagnostics designed to help combat infectious diseases and (inaccurate/inappropriate) antibiotic prescribing. Additionally, we agree that the choices made by end users of infectious disease and AMR diagnostics vary (inter)nationally.

\section{Compliance with Ethical Standards}

Funding No sources of funding were used to conduct this study or prepare this manuscript.

Conflict of interest J. P. Hays has no conflicts of interest directly relevant to this article.

\section{References}

1. Mott DJ, Hampson G, Llewelyn MJ, Mestre-Ferrandiz J, Hopkins MM. A multinational European study of patient preferences for novel diagnostics to manage antimicrobial resistance. Appl Health Econ Health Policy. 2020;18(1):69-79.

2. Kaman WE, Andrinopoulou ER, Hays JP. Perceptions of point-ofcare infectious disease testing among European medical personnel, point-of-care test kit manufacturers, and the general public. Patient Prefer Adher. 2013;7:559-77.

3. TEMPOtest-QC - CORDIS. [cited 2020 5th February]. https:// cordis.europa.eu/project/id/241742/reporting. Accessed 5 Feb 2020.

4. Kaman WE, Elshout G, Bindels PJE, Mitsakakis K, Hays JP. Current problems associated with the microbiological point-ofcare testing of respiratory tract infections in primary care. Future Microbiol. 2016;11(5):607-10.

5. Mitsakakis K, Kaman WE, Elshout G, Specht M, Hays JP. Challenges in identifying antibiotic resistance targets for pointof-care diagnostics in general practice. Future Microbiol. 2018;13(10):1157-64.

6. van Belkum A, Bachmann TT, Ludke G, Lisby JG, Kahlmeter G, Mohess A, et al. Developmental roadmap for antimicrobial susceptibility testing systems. Nat Rev Microbiol. 2019;17(1):51-62.

7. Hays JP, Mitsakakis K, Luz S, van Belkum A, Becker K, van den Bruel A, et al. The successful uptake and sustainability of rapid infectious disease and antimicrobial resistance point-of-care testing requires a complex 'mix-and-match' implementation package. Eur J Clin Microbiol Infect Dis. 2019;38(6):1015-22.

8. Hays JP. 'Out-of-the-box' thinking to help stop the spread of antibiotic resistance. Future Microbiol. 2016;11:995-8. 